\title{
Motivos de Eliminación en Competencias de Enduro Internacio- nal, Categoría Jinete Adulto, en Chile (2007-2014)
}

\author{
Reasons for Elimination in International Endurance Races, Adult Rider \\ Category, in ChILE (2007-2014) \\ Lisandro Muñoz-Alonzo ${ }^{1,3}$, Arturo Barrios Silva1, Jaime Cruces Leal', \\ Mario Briones Luengo ${ }^{2}$
}

\section{Resumen}

El objetivo del estudio fue determinar la frecuencia de los motivos de eliminación en competencias de enduro internacional (CEI) de 80-90, 120 y $160 \mathrm{~km}$, categoría adulto, realizadas en Chile en el periodo 2007-2014. La información fue tomada de la base de datos de la Federación Ecuestre Internacional, registrándose la distancia y número de equinos participantes, así como aquellos que finalizaron y aquellos que fueron eliminados de la carrera. En estos últimos se consideró los caballos retirados, descalificados y los que fallaron en calificar. Además, se registró la velocidad promedio de los ganadores para cada distancia. Se analizaron 99 carreras (42 de 80-90 km, 41 de $120 \mathrm{~km}$ y 16 de 160 $\mathrm{km})$, en las que participaron 919 caballos, donde 564 (61.4\%) finalizaron con éxito las carreras. El 3.2\% de los caballos fue descalificado, $4.1 \%$ retirado y $31.3 \%$ falló en calificar. De estos últimos, $25.4 \%$ fue eliminado por claudicación, $4 \%$ por razones metabólicas y $2 \%$ por otras razones. Se encontró asociación entre la distancia y el porcentaje de caballos que fallaron en calificar por claudicación $(\mathrm{p}<0.05)$ siendo mayor en carreras de $120 \mathrm{y}$ $160 \mathrm{~km}$. Asimismo, la velocidad promedio del ganador fue menor en carreras de mayor distancia ( $\mathrm{p}<0.001)$. Se concluye que en Chile, salvo el porcentaje de descalificación, el porcentaje total y de cada uno de los motivos de eliminación en CEI de 80-160 km es bajo.

Palabras clave: equino, claudicaciones, metabólico, larga distancia, carrera

\section{Abstract}

The aim of this study was to determine the frequency of reasons for elimination in international endurance competitions (IEC) of 80-90, 120 and $160 \mathrm{~km}$ in Chile in the period of 2007-2014. Records were obtained from the International Equestrian Federation database. Registered data included the distance and number of participating horses as well as those that successfully completed the race and those eliminated. In the latter

\footnotetext{
${ }^{1}$ Departamento de Ciencias Clínicas, ${ }^{2}$ Departamento de Ciencias Pecuarias, Facultad de Ciencias Veterinarias, Universidad de Concepción, Concepción, Chile

${ }^{3}$ E-mail: lismunoz@udec.cl
}

Recibido: 16 de setiembre de 2015

Aceptado para publicación: 9 de marzo de 2016 
case, data considered those that were retired, disqualified and failed to qualify. Also, average speed of winners for each distance was obtained. Ninety nine races were evaluated (42 in the range of 80-90 km, 41 in $120 \mathrm{~km}$, and 16 in $160 \mathrm{~km}$ ), with 919 participating horses, from whom $564(61.4 \%)$ completed the competition. There were $3.2 \%$ disqualified horses $4.1 \%$ retired and $31.3 \%$ failed to qualify. Within the later, $25.4 \%$ failed to qualify due to lameness, $4 \%$ for metabolic reasons and $2 \%$ for other reasons. Association was found between race distance and percentage of failed to qualify for lameness $(p<0.05)$, with a higher percentage in races of 120 and $160 \mathrm{~km}$. Moreover, the average speed of winners was lower in races of longer distances $(\mathrm{p}<0.001)$. It is concluded that in Chile, with the exception of the disqualification percentage, the overall and detailed percentage for elimination in IEC of $80-160 \mathrm{~km}$ is low.

Key words: equine, lameness, metabolic, long distance, race

\section{INTRODUCCIÓN}

El enduro es una competencia ecuestre contra reloj que implica recorrer entre $80 \mathrm{y}$ $160 \mathrm{~km}$ en un día, en la que se evalúa la habilidad atlética, resistencia y estado físico del equino en una determinada pista, terreno y clima (FEI, 2014). Es probablemente la disciplina atlética más demandante para los equinos (Harris y Schott, 2013). La competencia se divide en fases de 20 a $40 \mathrm{~km}$ cada una, donde se realiza una inspección veterinaria al final de cada fase (FEI, 2014). El objetivo de la inspección veterinaria es eliminar de la competencia a los animales cuyo bienestar esté amenazado y así protegerlo y evitar lesiones más serias (Nagy et al., 2012).

La inspección veterinaria incluye la evaluación de la condición general, temperatura rectal, frecuencia cardiaca, sistema respiratorio, estado metabólico (de acuerdo al color y humedad de las membranas mucosas, tiempo de relleno capilar, hidratación, actividad intestinal, índice de recuperación cardiaca y actitud), el desplazamiento (mediante la observación a distancia de un trote de $40 \mathrm{~m}$ ida y vuelta en línea recta) y la presencia de escoriaciones, laceraciones o heridas. Los caballos que no pasan exitosamente una inspección veterinaria son eliminados de la competencia y fallan en calificar para la siguiente fase. Además, existe la posibilidad de retiro voluntario del caballo por parte del jinete, siempre y cuando haya finalizado correctamente la fase en la cual partió y pasado exitosamente la inspección veterinaria correspondiente (FEI, 2016). Dentro de los factores de riesgo de eliminación en competencias de enduro se han reportado la distancia y el país (Nagy et al., 2014a).

En Chile, aun cuando la Federación Ecuestre Internacional (FEI) tiene registros de los resultados de las competencias de enduro internacionales (CEI) desde el año 2004, estos solo están completos desde el año 2007 (FEI, 2015). Por otro lado, no existen estudios al respecto en Chile, de allí que el objetivo de este estudio fue establecer la frecuencia de cada motivo de eliminación en equinos de enduro que participaron en las CEI de categoría jinete adulto en 80-90, 120 y 160 km realizados en Chile entre 2007 y 2014.

\section{Materiales y Métodos}

Se recolectó información en forma retrospectiva de la base de datos de la FEI. Se consideraron todos los caballos que participaron en las CEI de 80-90, 120 y $160 \mathrm{~km}$, categoría jinete adulto, realizados en Chile entre 2007 y 2014 (FEI, 2015). 
Se trabajó con información de 187 machos enteros, 467 machos castrados y 265 hembras, con edades entre 5 y 17 años. De cada carrera se registró el lugar, fecha, distancia y número de equinos participantes, así como aquellos que finalizaron con éxito la carrera y los eliminados. En estos últimos se encuentran los retirados, descalificados y los que fallaron en calificar por claudicación, razones metabólicas $\mathrm{u}$ otras razones (escoriaciones, laceraciones o heridas). Además, se registró la velocidad promedio de los caballos ganadores en kilómetros por hora $(\mathrm{km} / \mathrm{h})$

Se compararon las diferencias asociadas a la distancia con el porcentaje de finalización con éxito y con cada uno de los motivos de eliminación mediante una prueba de comparación de proporciones, con un nivel de significancia de $\mathrm{p}<0.05$. Los resultados de la variable velocidad del ganador para cada distancia son presentados como media, desviación estándar y rango. Para comparar la diferencia asociada a la distancia de la variable velocidad se utilizó un análisis de varianza y la prueba de Tukey, con un nivel de significancia de $\mathrm{p}<0.05$ (Daniel, 2009).

\section{Resultados}

Se analizaron 99 carreras con distancias de 80-90 km ( $\mathrm{n}=42), 120 \mathrm{~km}(\mathrm{n}=41)$ y $160 \mathrm{~km}(\mathrm{n}=16)$. Las carreras se llevaron a cabo en 16 pistas del país con la participación total de 919 caballos (61 a 182 participantes por año). En el Cuadro 1 se presentan las frecuencias de caballos que finalizaron con éxito las carreras, así como de los que fueron retirados, descalificados o fallaron en calificar por diversas razones. En el Cuadro 2 se muestra la velocidad promedio de los ganadores según las distancias de las competencias.

\section{Discusión}

El número total de caballos participantes en CEI en Chile es relativamente bajo si se le compara con países como los Emiratos Árabes Unidos (EAU) y Francia en 2011 donde participaron más de 1500 equinos o Uruguay y Estados Unidos de América (EEUU) donde ese mismo año hubieron 420 y 255 participantes, respectivamente (Nagy et al., 2014a). Entre las razones del bajo número de caballos participantes en CEI en Chile se pueden considerar la escasa cantidad de jinetes de enduro y la localización geográfica del país, ocasionando esto último mayores costos de traslado que limitan la participación de caballos de otros países. Una tercera razón podría ser que los premios o prestigio de las competencias realizadas en Chile no motivan a los jinetes, a diferencia de lo que ofrecen carreras como las que se llevan a cabo en EAU.

\section{Porcentaje de Finalización con Éxito}

El 61.4\% de caballos que finalizaron con éxito las carreras en Chile en el periodo en estudio fue ligeramente mayor al 58\% reportado por Ricard y Touvais (2007) en un estudio realizado en 9058 equinos en carreras de diferentes distancias en Francia y superior al $50.7 \%$ reportado por Nagy et al. (2014a) en un estudio realizado en 30741 equinos de 47 países que participaron en carreras de 100 a $160 \mathrm{~km}$ entre los años 2008 y 2011.

En relación a las carreras de $80 \mathrm{~km}$, el $67.8 \%$ de caballos que terminaron las carreras fue relativamente inferior al $75 \%$ indicado por Schott et al. (1997) en EEUU con 16 caballos, aunque este elevado valor pudo estar influenciado por el pequeño tamaño muestral, aun cuando en EEUU el porcentaje de finalización con éxito en otras categorías es también alto (Schott et al., 1997; Nagy et al., 2010). 
Cuadro 1. Número de caballos participantes en competencias de enduro internacional de categoría jinete adulto realizadas en Chile entre 2007 y 2014, así como las frecuencias de caballos que finalizaron con éxito, y fallaron en calificar, fueron retirados o descalificados

\begin{tabular}{|c|c|c|c|c|c|c|c|}
\hline \multirow{2}{*}{ 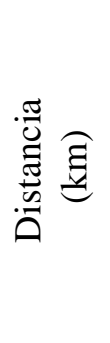 } & \multirow{2}{*}{ 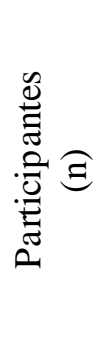 } & \multirow{2}{*}{ 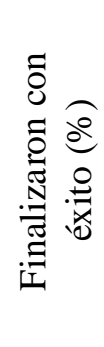 } & \multicolumn{3}{|c|}{$\begin{array}{c}\text { Fallaron en calificar } \\
\text { por }(\%)\end{array}$} & \multirow{2}{*}{ 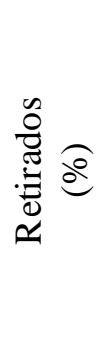 } & \multirow{2}{*}{ 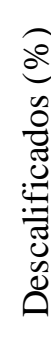 } \\
\hline & & & 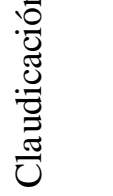 & 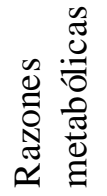 & 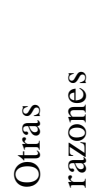 & & \\
\hline $80-90$ & 366 & $67.8^{\mathrm{a}}$ & $20.2^{\mathrm{a}}$ & 3.0 & 3.0 & 3.0 & 3.0 \\
\hline 120 & 444 & $58.3^{b}$ & $27.3^{\mathrm{b}}$ & 5.2 & 1.6 & 4.5 & 3.2 \\
\hline 160 & 109 & $52.3^{b}$ & $34.9^{\mathrm{b}}$ & 2.8 & 0 & 6.4 & 3.7 \\
\hline Total & 919 & 61.4 & 25.4 & 4.0 & 2.0 & 4.1 & 3.2 \\
\hline
\end{tabular}

${ }^{a, b}$ Superíndices diferentes dentro de columnas indican diferencia estadística $(p \varangle 0.05)$

Cuadro 2. Velocidad promedio de caballos ganadores en competencias de enduro internacional de 80-90, 120 y $160 \mathrm{~km}$ en Chile entre 2007 y 2014

\begin{tabular}{cccc}
\hline \multirow{2}{*}{$\begin{array}{c}\text { Distancia } \\
(\mathrm{km})\end{array}$} & $\begin{array}{c}\text { Ganadores } \\
(\mathrm{n})\end{array}$ & \multicolumn{2}{c}{$\begin{array}{c}\text { Velocidad promedio del caballo ganador } \\
(\mathrm{km} / \mathrm{h})\end{array}$} \\
\cline { 3 - 4 } & & Media \pm d.e. & Rango \\
\hline $80-90$ & 42 & $20.0 \pm 3.0^{\mathrm{a}}$ & $14.4-29.3$ \\
120 & 41 & $18.2 \pm 2.7^{\mathrm{b}}$ & $13.3-26.1$ \\
160 & 15 & $15.7 \pm 1.4^{\mathrm{c}}$ & $13.9-19.6$ \\
\hline
\end{tabular}

${ }^{a, b, c}$ Superíndices diferentes indican diferencia estadística $(p \varangle 0.001)$

Para las competencias de $120 \mathrm{~km}$, el $58.3 \%$ de finalización con éxito del presente estudio fue superior al obtenido en otros estudios con tamaños de muestra mayor a 1000 equinos donde no alcanzan el 50\% (Ricard y Touvais, 2007; Nagy et al., 2010). Asimismo, fue superior al $28.4 \%$ reportado para 67 equinos en Malasia (Lawan et al., 2012). La diferencia puede ser observada en países como Australia y EEUU, donde se registró porcentajes de finalización con éxito superio- res al 63\% en 2008 (Nagy et al., 2010). En competencias de $160 \mathrm{~km}$, el porcentaje de finalización con éxito en otros estudios varía entre 37.5 a 70\% (Schott et al., 1997, 2006; Robert et al., 2002; Barrey et al., 2006; Marlin et al., 2008; Barnes et al., 2010).

Es posible que el mayor porcentaje de caballos que finalizaron con éxito las carreras en Chile estuviera influenciado por las condiciones climáticas (Ecker y Lindinger, 
1995), las características del terreno (Ecker y Lindinger, 1995; Nagy et al., 2014b) y por una menor competitividad asociada al menor número de participantes por competencia (Nagy et al., 2014b).

\section{Porcentaje de Retiro}

El incremento en la frecuencia de retiro asociado a una mayor distancia de carrera, si bien no estadísticamente significativo, era esperado ya que el riesgo de lesiones aumenta con las distancias recorridas (Fielding et al., 2011; Nagy et al., 2014a). Se dispone de escasos estudios que reportan el porcentaje de retiro de caballos en CEI y ninguno hace referencia a competencias de $80 \mathrm{~km}$; no obstante, la información existente muestra un promedio de $7 \%$, con un rango de 0.7 a $11.9 \%$, dependiendo del país en que se realice la competencia (Barnes et al., 2010; Nagy et al., 2010, 2014a). Es así que el $4.1 \%$ de retiro total y de los promedios para cada distancia en Chile (Cuadro 1) fueron bajos, siendo solo superiores al 0.7 y $2.9 \%$ en 2008 en Australia y Uruguay, respectivamente, reportados por Nagy et al. (2010) para CEI iguales o mayores de $100 \mathrm{~km}$.

\section{Porcentaje de Descalificación}

El 3.2\% de descalificación fue alto comparado con el $0.4 \%$ reportado por Nagy et al. (2014a) en un estudio realizado en 47 países. Las causas de descalificación registradas en Chile fueron principalmente errores de registro de los jinetes y caballos al momento de la inscripción y la participación de caballos que no cumplían con el requisito de edad para la distancia. Esto implica una falta de atención al momento de la inscripción de los participantes y que debería ser fácilmente subsanada.

\section{Porcentaje de Falla en Calificar}

El porcentaje de caballos que fallaron en calificar en las competencias es considerado entre los médicos veterinarios de enduro como un indicador de bienestar animal (Nagy et al., 2010). El 31.3\% del presente estudio (26.2, 34.0 y $37.6 \%$ para las distancias de 80-90, 120 y $160 \mathrm{~km}$, respectivamente) están dentro de los rangos reportados en otros estudios, que va desde $18.9 \%$ hasta $71.6 \%$ (Schott et al., 1997; Barrey et al., 2006; Nagy et al., 2010; Lawan et al., 2012). En general, las mayores frecuencias de falla en calificar ocurren en países con condiciones climáticas más extremas como Malasia o EAU (Nagy et al., 2010; Lawan et al., 2012) o de mayor competitividad como EAU, Reino Unido y Uruguay, donde estos porcentajes son cercanos al 50\% (Nagy et al., 2010).

\section{Falla en calificar por claudicación}

La claudicación fue la principal causa de falla en calificar en Chile (25.4\%, 233/ 919), y coincide con otros resultados donde las frecuencias de falla en calificar por claudicación varían entre 8.9 a $56.6 \%$ (Schott et al., 1997; Schott et al., 2006; Muñoz et al., 2006; Ricard y Touvais, 2007; Barnes et al., 2010; Nagy et al., 2010, 2014a; Fielding et al., 2011; Lawan et al., 2012; Adamu et al., 2014). No obstante, es un porcentaje relativamente bajo ya que el promedio bordea el $30 \%$ en otros países (Nagy et al., 2014a). La mayor frecuencia de claudicación en relación a la mayor distancia de la carrera era esperable (Garlinghouse y Burrill, 1999; Fielding et al., 2011; Nagy et al., 2014a), ya que las injurias provocadas por la concusión y carga adicional de las articulaciones y tendones en las carreras de larga distancia tienen un efecto acumulativo (Lawan et al., 2012).

Además de la distancia, entre los factores de riesgo que inciden en la presentación de claudicaciones se encuentra la frecuencia de participación en competencias (Nagy et al., 2014b), las pistas (Ecker y Lindinger, 1995; Nagy et al., 2014b), los terrenos duros y ásperos (Lawan et al., 2012), el peso del equino (Garlinghouse y Burrill, 1999; Fielding et al., 2011), la conformación del animal, herrajes inadecuados (Lawan et al., 2012) y el número de participantes en las competencias (Nagy et al., 2010, 2014a). 


\section{Falla en calificar por razones metabólicas}

Las causas de falla en calificar por razones metabólicas están asociadas a la pérdida de electrolitos (Harris y Schott, 2013) y deficiente utilización de la glucosa sanguínea (Lawan et al., 2010), lo cual se manifiesta clínicamente con deshidratación severa (Adamu et al., 2014), fatiga, contracción sincrónica diafragmática, rabdomiolisis (Harris y Schott, 2013), arritmia cardiaca (Trigo et al., 2010), obstrucción esofágica (Fielding et al., 2009), diarrea (Fielding y Dechant, 2012), disminución de la motilidad intestinal (Adamu et al., 2014) y cólico (Fielding y Dechant, 2012).

El 4\% de falla en calificar por razones metabólicas en Chile fue relativamente bajo en comparación al rango de 3.2 a $53.7 \%$ reportado en otros estudios (Schott et al., 1997; Robert et al., 2002; Barrey et al., 2006; Muñoz et al., 2006; Schott et al. 2006; Ricard y Touvais, 2007; Nagy et al., 2010, 2014a,b; Trigo et al., 2010; Fielding et al., 2011; Lawan et al., 2012; Adamu et al., 2014). Un aspecto relevante en este estudio fue la similitud de las frecuencias de eliminación entre las distancias de las competencias; sin embargo, esto ha sido reportado en otros estudios (Ecker y Lindinger, 1995; Trigo et al., 2010; Fielding et al., 2011).

\section{Falla en calificar por otras razones}

Dentro de estas causas se encuentran lesiones traumáticas en piel y mucosas (FEI, 2014). La disminución en el porcentaje de falla en calificar por estas razones a medida que aumenta la distancia podría explicarse por el hecho que los caballos que participan en CEI de distancias mayores tienen mayor edad y número de competencias en su vida deportiva que les han permitido acostumbrar su piel y mucosas a los roces provocados por los aperos. Asimismo, los jinetes que participan en distancias mayores utilizan aperos de mejor calidad y tienen mayor experiencia.

\section{Velocidad Promedio}

La velocidad en CEI se encuentra influenciada por la distancia (Nagy et al., 2014a), el cansancio (Riber et al., 2006) y características genéticas (Ricard y Touvais, 2007). Además, por la presencia de enfermedades subclínicas, tales como el síndrome de úlcera gástrica equina (Nieto et al., 2004; Tamzali et al., 2011), problemas respiratorios (Frainpoint et al., 2011; Madariaga et al., 2014), y problemas cardiacos (Frainpoint et al., 2011).

No se dispone de estudios previos en relación a la velocidad promedio del ganador en distancias de $80-90 \mathrm{~km}$. La velocidad media de los caballos ganadores en CEI de 120 $\mathrm{km}$ en Chile estuvo dentro del rango reportado en CEI de 100 a $120 \mathrm{~km}$ que va de $14.1 \mathrm{a}$ $26.2 \mathrm{~km} / \mathrm{h}$ (Nagy et al., 2010); sin embargo, llama la atención que fuera superior a lo reportado el año 2008 en diversos países donde en esas carreras hubo mayor número de participantes (Nagy et al., 2010). De hecho, en el estudio de Nagy et al. (2014a) realizado en 47 países, se reporta una velocidad media de los caballos ganadores de $17.5 \mathrm{~km} / \mathrm{h}$, pudiendo indicarse que el $18.2 \mathrm{~km} / \mathrm{h}$ de velocidad media de los caballos ganadores en Chile sería muy similar al obtenido en la mayoría de los países.

En forma similar, la velocidad media de los caballos ganadores en CEI de $160 \mathrm{~km}$ en Chile $(15.7 \mathrm{~km} / \mathrm{h})$ fue cercana al promedio de $16.3 \mathrm{~km} / \mathrm{h}$ reportado por Nagy et al. (2014a) en su estudio realizado en 47 países.

\section{Conclusiones}

- Entre los 919 participantes en 99 carreras en competencias de enduro internacionales en Chile en distancias de 80-90, 120 y $160 \mathrm{~km}$ entre 2007 y 2014 , el $61.4 \%$ de caballos participantes finalizaron con éxito las carreras. Asimismo, el 33.4\% de los caballos fallaron en calificar, $4.1 \%$ 
fueron retirados y $3.2 \%$ fueron descalificados.

- La mayor causa de eliminación fue falla en calificar por claudicación (25.4\%, 233/919), siendo menor en distancias de $80-90 \mathrm{~km}(20.2 \%)$ que en distancias mayores (27.3 y $34.9 \%$ en distancias de 120 y $160 \mathrm{~km}$, respectivamente $)(\mathrm{p}<0.05)$.

\section{Literatura Citada}

1. Adamu L, Noraniza MA, Rasedee A, Bashir A. 2014. Physical parameters and risk factors associated with elimination of Arabian and crossed Arabian endurance horses during a 120$\mathrm{km}$ endurance race. J Equine Vet Sci 34: 494-499. doi: 10.1016/j.jevs.2013.10.175

2. Alexander GR, Haines GR. 2012. Surgical colic in racing endurance horses. Equine Vet Educ 24: 193-199. doi: 10.1111/j.2042-3292.2011.00360.x

3. Barnes A, Kingston J, Beetson S, Kuiper C. 2010. Endurance veterinarians detect physiologically compromises horses in a $160 \mathrm{~km}$ ride. Equine Vet J 42 (Suppl 38): 6-11. doi: 10.1111/j.20423306.2010.00225.x

4. Barrey E, Muncher E, Robert C, Amiot F, Gidrol X. 2006. Gene expression profiling in blood cells of endurance horses completing competition or disqualified due to metabolic disorder. Equine Vet J (Suppl 36): 43-49.

5. Daniel WW, Cross CL. 2013. Biostatistic. A foundation for analysis in the health sciences. $10^{\text {th }}$ ed. Hoboken: Wiley. $960 \mathrm{p}$.

6. Ecker GL, Lindinger MI. 1995. Effects of terrain, speed, temperature and distance on water and ion losses. Equine Vet J 27: 298-305. doi: 10.1111/ j.2042-3306.1995.tb04941.x

7. FEI [Fédération Equestre Internationale]. 2015. FEI Database. [Internet]. Disponible en: https:// data.fei.org/Calendar/search.aspx
8. FEI [Fédération Equestre Internationale]. 2016. Endurance rules. $9^{\text {th }}$ ed. Lausanne: FEI. [Internet]. Disponible en: www.fei.org/sites/default/Endurance Rules_2016 clean_0.pdf

9. Fielding CL, Dechant JE. 2012. Colic in competing endurance horses presenting to referral centres: 36 cases. Equine Vet J 44: 472-475. doi: 10.1111/ j.2042-3306.2011.00462.x

10. Fielding CL, Magdesian G, Rhodes DM, Meier CA, Higgins JC. 2009. Clinical and biochemical abnormalities in endurance horses eliminated from competition for medical complications and requiring emergency medical treatment: 30 cases (2005-2006). J Vet Emerg Crit Care 19: 473-478. doi: 10.1111/j.1476-4431.2009.00441.x

11. Fielding CL, Meier CA, Balch OK, Kass PH. 2011. Risk factors of the elimination of endurance horses from competition. J Am Vet Med Assoc 239: 493-498. doi: 10.2460/javma.239.4.493

12. Fraipoint A, van Erck $E$, Ramery $E$, Richard E, Denoix J-M, Lekeux P, Art T. 2011. Subclinical diseases underlying poor performance in endurance horses: diagnostic methods and predictive tests. Vet Rec 169: 154. doi: 10.1136/vr.d4142

13. Garlinghouse SE, Burrill MJ. 1999. Relationship of body condition score to completion rate during $160 \mathrm{~km}$ endurance races. Equine Vet J (Suppl. 30): 591-595.

14. Harris PA, Schott HC II. 2013. Nutritional management of elite endurance horses. In: Geor RJ, Harris PA, Coenen M (eds). Equine applied and clinical nutrition. Equine welfare and performance. Edinburgh: Saunders Elsevier. p 272-288.

15. Lawan A, Noraniza MA, Rasedee A, Bashir A. 2010. Effects of race distance on physical, hematological and biochemical parameters of endurance horses. Am J Anim Vet Sci 5: 244-248. 
16. Lawan A, Noraniza MA, Rasedee A, Bashir A. 2012. Prevalence of lameness and metabolic disorders in endurance horses. Malaysian J Vet Res 3: 33-37.

17. Madariaga C, Goic M, Hernández A. 2014. Descripción de patologías respiratorias superiores, mediante endoscopía, en 33 caballos de enduro, post competencia. En: XVIII Congreso Chileno de Medicina Veterinaria. Santiago de Chile: Asociación de Facultades y Escuelas de Veterinaria del Consejo de Rectores.

18. Marlin DJ, McEwen J, Sluyter F. 2008. Completion and treatment rates in modern endurance racing. In: IV International Equitation Science Conference. Dublin: International Society for Equitation Science.

19. Muñoz A, Cuesta I, Riber C, Gata J, Trigo P, Castejón FM. 2006. Trot asymmetry in relation to physical performance and metabolism in equine endurance rides. Equine Vet J 38: 5054. doi: 10.1111/j.2042-3306.2006. tb05512.x

20. Nagy A, Murray JK, Dyson S. 2010. Elimination from elite endurance rides in nine countries: a preliminary study. Equine Vet J 42 (Suppl. 38): 637-643. doi: 10.1111/j.2042-3306.2010.00220.x

21. Nagy A, Dyson S. Murray JK. 2012. A veterinary review of endurance riding as an international competitive sport. Vet J 194: 288-293. doi: 10.1016/j.tvj1.2012. 06.022.

22. Nagy A, Murray JK, Dyson S. $2014 a$. Descriptive epidemiology and risk factors for eliminations from Fédération Equestre Internationale endurance rides due to lameness and metabolic reasons (2008-2011). Equine Vet J 46: 38-44. doi: 10.1111/evj.12069

23. Nagy A, Murray JK, Dyson S. $2014 b$. Horse-, rider-, venue- and environment risk factors for elimination from Fédération Equestre Internationale endurance rides due to lameness and metabolic reasons. Equine Vet J 46: 294299. doi: $10.1111 /$ evj. 12170

24. Nieto JE, Snyder JR, Beldomenico P, Aleman M, Kerr JW, Spier SJ. 2004. Prevalence of gastric ulcers in endurance horses. A preliminary report. Vet J 167: 33-37. doi: 10.1016/j.tvjl.2003.09.005

25. Riber C, Cuesta I, Muñoz A, Gata J, Trigo P, Castejón FM. 2006. Equine locomotor analysis on vet-gates in endurance events. Equine Vet J 38 (Suppl 36): 55-59. doi: 10.1111/j.20423306.2006.tb05513.x

26. Ricard A, Touvais M. 2007. Genetic parameters of performance trait in horse endurance races. Livest Sci 110: 118125. doi: 10.1016/j.livsci.2006.10.008

27. Robert C, Benamou-Smith A, Leclerc J-L. 2002. Use of the recovery check in long-distance endurance rides. Equine Vet J (Suppl. 34): 106-111.

28. Schott HCII, Marlin DJ, Geor RJ, Holbrook TC, Deaton CM, Vincent T, Dacre K, Schroter RC, JoseCunilleras E, Cornelisse CJ. 2006. Changes in selected physiological and laboratory measurement in elite horses competing in a $160 \mathrm{~km}$ endurance ride. Equine Vet J (Suppl 36): 37-42.

29. Schott HCII, McGlade KS, Molander HA, Leroux AJ, Hines MT. 1997. Body weight, fluid, electrolyte, and hormonal changes in horses competing in 50- and 100-mile endurance rides. Am J Vet Res 58: 303-309.

30. Tamzali Y, Marguet C, Priymenko N, Lyazrhi F. 2011. Prevalence of gastric ulcer syndrome in high-level endurance horses. Equine Vet J 43: 141-144.

31. Trigo P, Castejón F, Riber C, Muñoz A. 2010. Use of biochemical parameters to predict metabolic elimination in endurance rides. Equine Vet J 42(Suppl. 38): $142-146$. 